\title{
THE EARTH'S VARIABLE ROTATION: SOME GEOPHYSICAL CAUSES
}

\author{
Kurt Lambeck \\ Research School of Earth Sciences \\ The Australian National University \\ P.O. Box 4 \\ Canberra A.C.T. 2601
}

\section{Introduction}

The Earth's variable rotation, its departures from what it would be if it were a rigid body rotating in isolation, has occupied the interest of astronomers and geophysicists for more than 100 years. The reason for this is quite clear when one becomes aware of the range of processes that perturb the Earth from uniform rotation (Figure 1). A complete understanding of the driving mechanisms requires a study of the deformation of the solid Earth, of fluid motions in the core and the magnetic field, of the mass redistributions and motions within the oceans and atmosphere, and of the interactions between the solid and fluid regions. The discussion of evidence for the variable rotation includes the examination of not only a variety of optical telescope evidence that goes back some 300 years, but also of historical records of lunar and solar eclipses, and planetary occultations and conjunctions for perhaps the past three millenia. The geological record, in the form of fossil growth rhythms in organisms such as corals, bivals or brachiopods or as cyclic organic growth and sediment sequences such as stromatolites or banded iron formations, extend, albeit with considerable uncertainty, the record back through Phanerozoic time and into the Early Precambrian. To this variety of measurement techniques now has to be added the new methods derived from the space-oriented technological developments of the past few decades.

The geophysical studies of the Earth's rotation have their roots in the works of Lord Kelvin, Sir George Howard Darwin and Sir Harold Jeffreys amongst others. Walter Munk and Gordon MacDonald thoroughly reviewed the subject in their monograph and this work has dominated the subject ever since (Munk and MacDonald, 1960). Even now there are few aspects of the subject that does not bear their imprint. Since the publication of their book in 1960 there have been significant developments of both an observational and interpretative nature. The introduction of atomic time and the improvement of the determination of universal time led to progress in our knowledge, if not necessarily understanding, of the high frequency variations in the length of day. The revised pole positions from 1900 onwards led to better estimates, although still much debated ones, of the Chandler wobble parameters. The reinterpretation of the eclipse data has led away from the classical records to more reliable historical documents. But curiously enough the now favoured answers are not very different from the early results. 


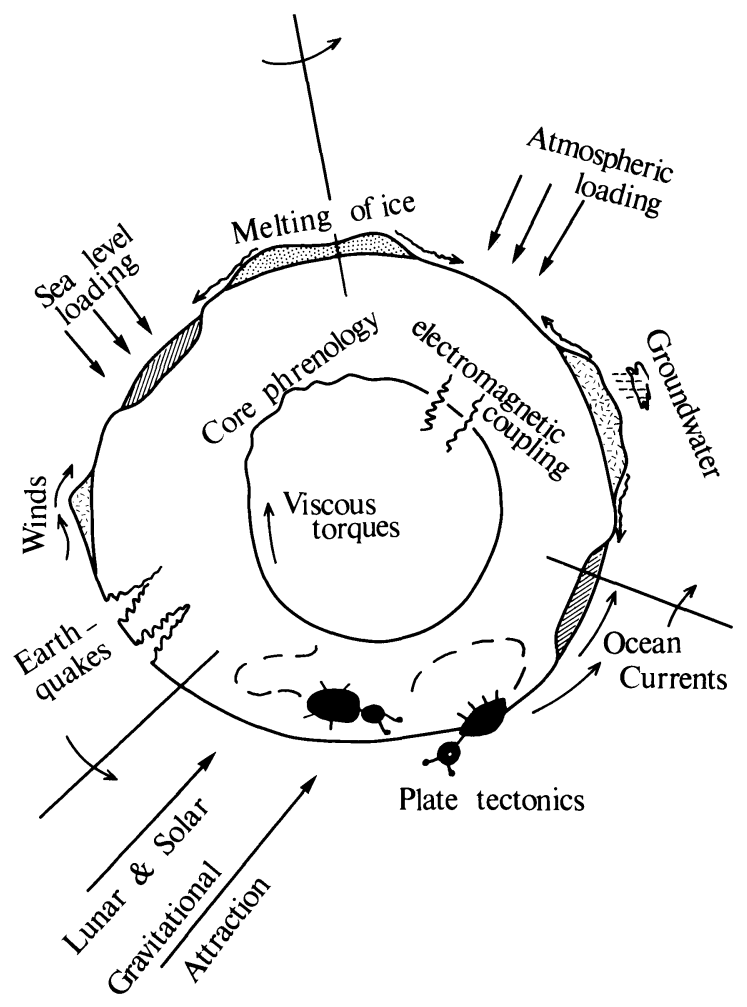

Figure 1. Schematic illustration of the forces that perturb the Earth's rotation (from Lambeck, Nature 286, p. 104, 1980). The core phrenology refers to $R$. Hide's model of topographic coupling. The beetles refer to T. Gold's representation of continental drift.

The palaentological evidence has raised the possibility of extending the record of the Earth's rotation beyond the 1 millionth of the age of the earth that was the case to about $10 \%$ and possibly longer. These observations need, however, to be viewed with a healthy scepticism. Also important are the major developments that have occurred in solid-earth physics: improved radially symmetric models of the planet; improved theories for the response to surface loading; the recognition of the major far-field displacements that occur in association with large earthquakes; and the development of the plate tectonics hypothesis. Improved descriptions of motions in the oceans and atmosphere have also made a significant contribution to understanding aspects of the Earth's rotation.

The developments up to the late 1970's were examined and reviewed by myself in 1980 (Lambeck, 1980). By that time, developments in space science and 
technology were spurring new interest in the subject of the Earth's rotation. Precise tracking of satellites for gravity field studies, laser ranging for studying lunar motion, long-baseline radio interferometric observations for deciphering extra-galactic radio sources, and the precise manoeuvring of interplanetary flights all require an equally precise tracking of the motions of the Earth's rotation axis. At the same time, these new techniques permit the changes in rotatation to be measured with an unprecedented precision and resolution. The geophysicist's signal is astrophysicist's and space engineer's noise. The most notable achievement by that time had been the determination of polar motion from the Doppler tracking of satellites. By 1980 the impact of these new methods on the geophysical discussion had been minimal but the results were sufficiently promising to anticipate that new excitation functions would rise out above the measurement noise level, even if this signal may itself be largely "meteorological" noise.

How has the subject evolved since 1980? It is probably too short a time, barely six Chandler wobble periods, to expect substantive developments that can be attributed solely to the result of the new methods. Much of the present discussion will, therefore, still be based on the older observational data: an appropriate reminder that this earlier data will remain important for many decades to come. Brief reviews, in which some of this progress is also discussed, are by Merriam (1983), Lambeck (1983) Rochester (1984), and Wahr (1985).

\section{Excitation Functions}

The fundamental equations describing the rotation of a non-rigid planet is the Eulerian equation in which both the angular momentum of the planet and the torques $\mathbf{L}$ acting on it are time dependant. These equations are sometimes referred to as the Liouville equations. In most discussions of non-rigid rotation, the excursions from rigid body rotation are small and a perturbation form of the equations suffice. If the conventional terrestrial system, an "earth-fixed" cartesian coordinate system $x_{i}(i=1,2,3)$, is introduced in which $x_{3}$ lies close to the mean position of the rotation axis, it is convenient to define the rotation of these axes about themselves as

$$
\omega_{1}=\omega_{0} \mathrm{~m}_{1} ; \quad \omega_{2}=\omega_{0} \mathrm{~m}_{2} ; \quad \omega_{3}=\omega_{0}\left(1+\mathrm{m}_{3}\right)
$$

where $\omega_{0}$ is the mean angular velocity of the Earth. The $m_{i}$ are small dimensionless quantities, of the order $10^{-6}$ or less. The $m_{1}, m_{2}, 1+m_{3}$ represent the direction cosines of $\omega$ relative to $x_{3} . m_{1}$ and $m_{2}$ specify the position of the instantaneous rotation axis, the polar motion. $m_{3}$ represents departures in the speed of rotation from the uniform value of $\omega_{0}$. The change in length-of-day, $\Delta($ l.o.d $)$ is defined as $m_{3}=-\Delta($ l.o.d $) /($ l.o.d $)$. The equations of rotation then take the form

where

$$
\begin{aligned}
\mathrm{j} \dot{\mathbf{m}} / \sigma_{\mathrm{r}}+\mathbf{m} & =\psi \\
\mathrm{m}_{3} & =\psi_{3},
\end{aligned}
$$

$$
\begin{aligned}
& \mathbf{m}=\mathrm{m}_{1}+\mathbf{j} \mathrm{m}_{2} \\
& \psi=\psi_{1}+\mathrm{j} \psi_{2} \\
& \sigma_{\mathrm{r}}=\omega_{0}(\mathrm{C}-\mathrm{A}) / \mathrm{A} .
\end{aligned}
$$

$\mathrm{C}$ and $\mathrm{A}$ are the mean polar and equatorial moments of inertia. The $\psi_{\mathrm{i}}$ are the excitation functions, characterizing the torques, relative motions and inertia tensor 
changes. They can be written as

with,

$$
\psi_{\mathrm{i}}=\psi_{\mathrm{i}}(\text { torque })+\psi_{\mathrm{i}}(\text { matter })+\psi_{\mathrm{i}}(\text { motion })
$$

and

$$
\begin{aligned}
& \psi(\text { torque })=\mathrm{j} \mathrm{L} / \omega_{0}^{2}(\mathrm{C}-\mathrm{A}) \\
& \psi(\text { matter })=\Delta \mathrm{I} /(\mathrm{C}-\mathrm{A}) \\
& \psi(\text { motion })=\left[\omega_{0} h-\mathrm{j} \Delta \mathrm{I}-\mathrm{jh}\right] / \omega_{0}^{2}(\mathrm{C}-\mathrm{A}),
\end{aligned}
$$

The inertia tensor $I_{i j}$ of the Earth is written as

$$
\begin{array}{ll}
I_{11}=A+\Delta I_{11}(t), & I_{22}=A+\Delta I_{22}(t), \\
I_{33}=C+\Delta I_{33}(t), & I_{i j}=\Delta I_{i j} \quad(i \neq j)
\end{array}
$$

where the time dependent perturbations $\Delta \mathrm{I}_{\mathrm{ij}}$ are assumed to be small. Also,

$$
\Delta \mathrm{I}=\Delta \mathrm{I}_{13}+\mathrm{j} \Delta \mathrm{I}_{23} ; \mathrm{h}=\mathrm{h}_{1}+\mathrm{jh}_{2}
$$

The $h_{i}$ are the angular momentum terms resulting from any motion of particles relative to the $x_{i}$ axes. Equations 2 define the motion of the rotation axis relative to the earth-fixed axes $x_{i}$. These equations clearly separate the geodetic astronomical problem of measuring the rotation (the left-hand-side of 2) from the geophysical problem of evaluating the mechanisms that drive the variable rotation, the excitation functions $\psi_{\mathrm{i}}$. These equations also separate the polar motion (equation $2 \mathrm{a}$ and the definition $5 \mathrm{a}$ ) from the length-of-day changes (equations $2 \mathrm{~b}$ and the definition 5b), a separation that is convenient in view of different observational techniques that have been used, to determine the two components of the rotational motion. The above equations are generally valid, provided that the excursions of the instantaneous rotation axis from its mean position are small. Otherwise, a more complete description is required in which the squares and products of small quantities must be retained but now the equations lose much of their convenience. At the present levels of accuracy of both the geodetic and geophysical data the first order equations, modified if necessary to include the small ellipticity of the polar motion path resulting from the axial asymetry of the Earth's density distribution $\left(I_{11} \neq I_{22}\right)$, largely suffice. Equation (2) defines one part of the rotation problem. The other part is the motion of either the rotation axis or the earth-fixed axes in space. This latter motion can be expressed in several ways, for example by Euler's kinematic equations. If we define an inertial reference frame $X_{i}$, the position of $x_{i}$ relative to it can be defined by the Euler angles

$$
\begin{aligned}
& \alpha_{1}=\text { inclination of the } x_{1}-x_{2} \text { plane on the mean ecliptic, } \\
& \alpha_{2}=\text { angle in the } x_{1}-x_{2} \text { plane between } x_{1} \text { and the ascending node of } \\
& \text { the } x_{1}-x_{2} \text { plane on the ecliptic, } \\
& \alpha_{3}=\text { angle in the } x_{1}-x_{2} \text { plane between the ascending node and the } x_{1} \\
& \text { axis. }
\end{aligned}
$$

The Euler equations then are 


$$
\left[\begin{array}{ll}
\alpha_{1} & \\
\sin \alpha & \dot{\alpha}_{2} \\
\dot{\alpha}_{3}+\dot{\alpha}_{2} \cos \alpha_{1}
\end{array}\right]=\left[\begin{array}{ccc}
\cos \alpha_{3} & -\sin \alpha_{3} & 0 \\
\sin \alpha_{3} & \cos \alpha_{3} & 0 \\
0 & 0 & 0
\end{array}\right] \quad\left[\begin{array}{l}
\omega_{1} \\
\omega_{2} \\
\omega_{3}
\end{array}\right]
$$

and determine the motion of $x_{i}$ relative to $X_{i}$, once the dynamic equations have been solved for the $\omega_{i}$. In equation (7), the angle $\alpha_{3}$ is equal to $\omega_{3}$ t and has a nearly diurnal period. This means that some nearly diurnal terms in the solutions for $\omega_{i}$, generally of very small amplitude such as the lunar and solar torques on the earth, become the major terms in the Eulerian angles $\alpha_{i}$. Large-amplitude long-period terms in the solutions for $\omega_{i}$, such as the Chandler wobble, make only very minor contributions to the $\alpha_{i}$. Certain geophysical quantities are therefore best studied by examining the $\mathrm{m}_{\mathrm{i}}$ (the polar motion and the changes in length-of-day), while others are best studied by examining the $\alpha_{i}$ (the precession and nutation), always assuming that both astronomical quantities can be measured with comparable precision. In either case, the geophysical problem is one of evaluating the excitation functions; the variations with time of the torques $\mathrm{L}$ acting on the Earth, of the changes in the inertia tensor $\Delta \mathrm{I}$, and of the momentum of motions of particles making up the Earth relative to the earth-fixed axes. An obvious, yet important point is that these excitations are integral quantities over the whole volume or surface of the Earth. The Earth's response is a globally integrated one to what may be either localized or global excitation functions. One example of this is the periodic change in the mass distribution within the ocean caused by the tides. The spatial spectrum of these changes is very broad, yet the Earth's rotation responds only to the small amplitude second degree component in the tide expansion. As such, the geophysical constraints imposed by the rotational data are of ten only of restricted value in understanding the process themselves.

\section{The rotation spectra}

Figure 2 illustrates schematically the polar motion based on some 80 years of telescope observations of the variation in astronomical latitude. The spectrum is dominated by the Chandler wobble peak and the annual oscillation. The former represents a free oscillation of the Earth and is associated with three main problems: (i) Can its period be quantitatively explained? (ii) Because any free oscillation in physics will eventually be damped, how is this oscillation maintained or excited in the presence of dissipation? (iii) Where is the rotational energy dissipated? The search for answers to these questions involves excursions into the physics of the core, mantle, oceans and atmosphere.

In 1980 the answer to these questions would have been as follows (Lambeck,1980). The period of the Chandler wobble is adequately explained; this period is not particularly sensitive to the fine detail of the radial structure of the mantle and neither is the detailed core structure very significant unless the core is strongly stably stratified. The lengthening of the period by the departure of the ocean from equilibrium theory does not appear to be important but that caused by mantle inelasticity does, amounting to several days depending on the $Q$ structure. The accuracy of the observed period of the wobble was inadequate to constrain tightly models of mantle anelasticity. The 1980 response to the question of excitation of the wobble was that the nature of the mechanism remained obscure. 


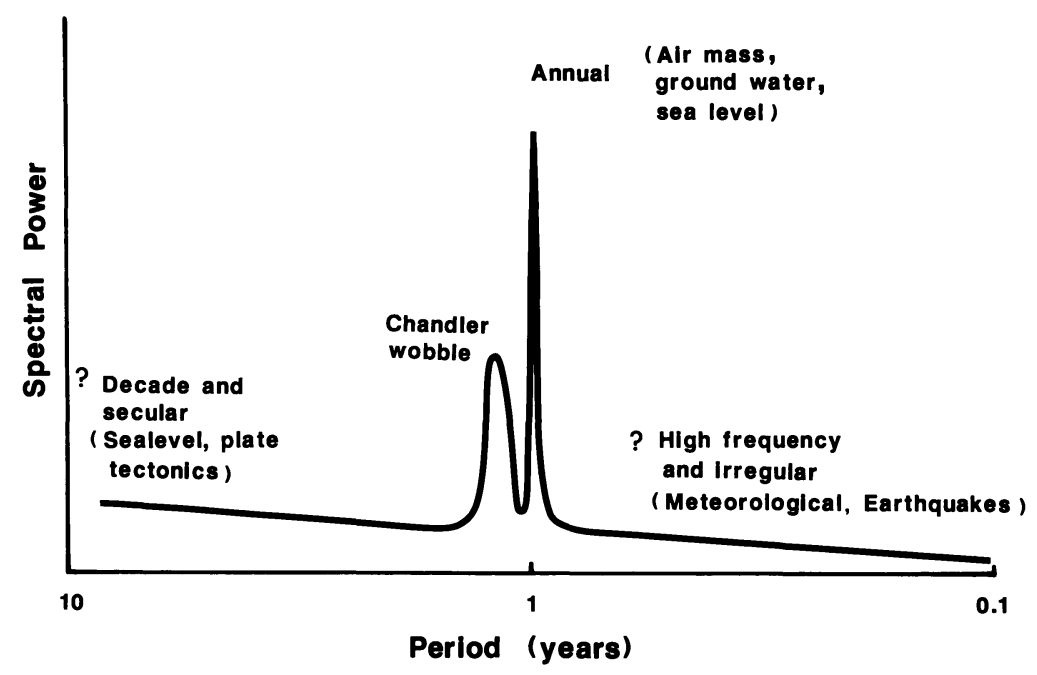

Figure 2. Schematic spectrum of the Earth's polar motion and possible excitation mechanisms.

Neither seismic nor atmospheric mechanisms appeared adequate on their own but a combination of seismic, aseismic and meteorological factors could possibly maintain the wobble. The question of dissipation of the Chandler wobble energy also remained elusive. The likely sink was the ocean but this is difficult to test because of the very small amplitude of the pole tide (a few millimeters) in the open oceans.

The second peak in the polar motion spectrum is the annual oscillation. This is a forced wobble that is driven by the seasonal redistributions of mass within and between the oceans, atmosphere and ground and surface waters. In 1980 the greatest uncertainty in evaluating this excitation function was the ground and surface water term, being based on quite inadequate hydrological models and observations.

The polar motion spectrum contains little power at the other frequencies that can be unequivocally attributed to anything but measurement noise. Any semi-annual term is small, as would be expected from the evaluation of the meteorological excitation spectrum. "Decade" fluctuations have been suggested but the evidence for this remains tenuous because of the inhomogeneity of the data sets. The small secular drift of the mean pole is perhaps more significant, the pole moving in a generally westward direction at a rate of $0 \cdot 002-0 . " 003$ per year. This is attributable to the delayed response of the Earth to the late Pleistocene 
melting of the polar ice caps time (Nakiboglu and Lambeck, 1980; Yuen et al. 1982; Wu and Peltier, 1984). If this interpretation is correct, it provides a useful additional constraint on the mantle viscosity.

The length-of-day spectrum has more character (Figure 3). This spectrum is based on a variety of data; from the telescopic observations that go back to the seventeenth century and which are referenced to ephemeris time, to the astrolabe and photozenith telescope observations which, since 1955, are referenced to the atomic time scale. These observations exhibit a major low frequency or secular component: the length-of-day is increasing at a rate of about 1-2 milliseconds (ms) every century. Superimposed upon this are the decade fluctuations, a loosely defined period that encompasses variations on time scales of several decades to perhaps 5 years. Changes in length-of-day of some 4-5 ms have occurred within 10-30 years on several occasions since the record became sufficiently reliable in the mid-to-late nineteenth century. Meteorologically driven changes in the length-of-day include the seasonal terms of 12 and 6 months as well as a somewhat enigmatic biennial term. Periodic tidal deformations rise above the measurement and meteorological noise levels near 27 and 14 days.

The important questions that arise from the length-of-day spectrum include the following: What is the cause of the secular increase in the length-of-day? Where is the rotational energy lost by this secular change being dissipated? What is the validity of extrapolating these secular changes beyond the observational record to infer changes to the Earth's rotation over geological time scales. The 1980 answers to these questions would differ little from today's; that tidal dissipation is the main cause for the secular change in the length-of-day; that at least $95 \%$ of the energy is dissipated in the oceans, although the mechanism by which this energy is lost in the ocean remains obscure. Extrapolation of the secular change beyond the observational record, including the eclipse record extending to about 1000 years $\mathrm{BC}$, is sufficiently uncertain to prevent strong conclusions to be drawn about the Earth-Moon configurations in the geological past. In this context a recent interesting observation is by Walker and Zahnle (1986) who suggest that periodicities in banded iron formations can be used to constrain the Earth-Moon geometry in Precambrian time but other interpretations may be equally plausible (Lambeck, 1986).

The nature of the decade fluctuations was also unclear in 1980. Only the core is sufficiently mobile and has sufficient inertia to explain these fluctuations but the examination of mechanisms by which these motions are transferred to the mantle remained one of a process of elimination by which the least well constrained mechanism, electromagnetic coupling, also becomes the most plausible. A curiosity raised by these decade changes is that they appear to follow some changes in climate but a specific coupling mechanism had not been offered.

The seasonal changes in the length-of-day were almost wholly attributed to zonal winds, the angular momentum of the atmosphere does not remain constant but part of it is exchanged with the solid Earth. Variations in the Earth's rotation at higher frequencies were also attributed to zonal atmospheric circulation patterns (near 8 months, 40-55 days, near the tidal terms, and at irregular periods as short as a few days; Lambeck and Cazenave, 1977; Lambeck, 1980). This high-frequency meteorological noise has important consequences upon the discussion of the geophysical factors perturbing the Earth's rotation. It masks other irregular short-duration excitations, it contaminates the tidal signals near 27 and 13 days, and it masks the decade changes in length of day. Thus if further insights into the global dynamics of the solid Earth are sought from observations of the Earth's 


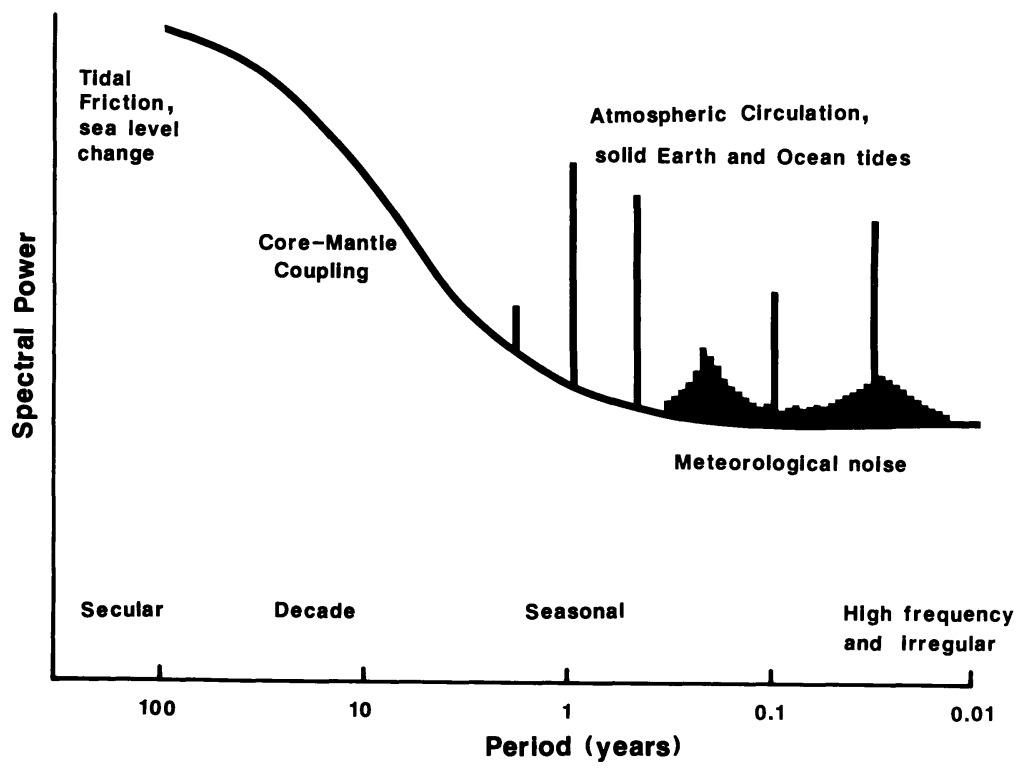

Figure 3. Schematic spectrum of the Earth's changes in length-of-day or in rotational velocity and possible excitation mechanisms.

rotation, it becomes also necessary to evaluate the meteorological excitation functions with comparable accuracy and resolution.

\section{The Chandler Wobble}

Wobble period. Analyses of the polar motion data have established a Chandler wobble period of about 434-436 sidereal days (Wilson and Vicente, 1980; Okubo, 1982a) similar to previous analyses (e.g. Jeffreys, 1968). Two old concepts about the Chandler wobble period have recently been revived. Carter (1982) suggested that the Chandler wobble period is time variant (see for example, Proverbio et al., 1972) while Chao (1983) suggested that the Chandler peak comprises two nearly equal oscillations separated in frequency by about 0.02 cycle $\mathrm{yr}^{-1}$ (see also, Colombo and Shapiro, 1968). Okubo (1982a) (see also Pederson and Rochester, 1972) confirmed, however, that these interpretations are artefacts of the methods of spectral analysis used and that the model of a single oscillator, subjected to random excitation as well as damping, is consistent with the data.

Model periods for an elastic Earth, fluid core, and equilibrium ocean have been estimated by Smith and Dahlen (1981) to be $426.7 \pm 0.5$ sidereal days, the uncertainty resulting mainly from uncertainties in the earth models. This result is 
for a neutrally stratified core (the Brunt-Vaisala frequency $\mathrm{N}$ is zero). A stably stratified core will lead to an increase in the period by up to 2 days for $\mathrm{N}^{2} \simeq 3.4 \times 10^{-7}$ (Smith, 1977) but the seismic evidence is consistent with $\mathrm{N}=0$ (Masters, 1979) and any uncertainty in the wobble period arising from this source is less than 0.2 days. Smith and Dahlen also suggest that their linearized normal mode theory will not introduce errors greater than about \pm 0.3 days. Recent estimates of the lengthening of the period by the equilibrium ocean on an Earth model comprising an elastic mantle and fluid core differ by about 1 day: Smith and Dahlen obtain 29.8 sidereal days and Dickman and Steinberg (1986) obtain 30.9 days. The major omissions in the above estimate for the wobble period of 426.7 days are believed to be two-fold; the neglect of dissipation of wobble energy in the solid Earth and the departure of the ocean pole tide from equilibrium. Both have the effect of lengthening the theoretical or model period, bringing it into closer agreement with the observed value of about 434-436 days. Coupling of the core motions to the mantle by other than pressure forces, are believed to be unimportant.

Does the pole tide follow an equilibrium theory? This question is of obvious importance in understanding the Chandler wobble period but it is also of interest in understanding the dynamics of the oceans at long periods. Models, of various degree of approximation, do not indicate that this tide will depart sufficiently from equilibrium to affect the wobble period in any significant way (Carton and Wahr, 1983; Dickman, 1985; O'Connor, 1986). Dickman considers that the lengthening of the period is at most 1 day while O'Connor estimates that it is only a fraction of a day, a conclusion previously reached by Lambeck (1980, p.202). It is therefore tempting to associate the difference between the observed and predicted periods, about 7-9 days, to the lengthening effect of the anelasticity of the mantle since simple models indicate that this may amount to several days, particularly if the mantle $Q$ is frequency dependent (see page 202 of Lambeck, 1980). Better models of this period lengthening are discussed by Smith and Dahlen (1981) who suggest that the shear $Q$ is proportional to (frequency) ${ }^{\alpha}$ with $\alpha$ in the range $0.09-0.15$.

Whatever the $Q$ value will finally turn out to be, this estimate of the frequency dependence is important in a geophysical context (see, for example, Jeffreys, 1970; Anderson and Minster, 1979). Considerable information on the Q is available at seismic frequencies down to about 1 cycle/hour. Diurnal and semi-diurnal tidal estimates of the solid Earth $Q$ are uncertain because of contamination by the ocean tide and the validity of extrapolation dissipation models to the low tidal frequencies is difficult to test.

Excitation. Sir Harold Jeffries has on several occasions emphasized that the estimation of dissipation and excitation parameters are closely linked; that dissipation constants are a function of the assumptions made about the nature of the excitation process (e.g. Jeffreys, 1940). In the presence of dissipation, the Chandler wobble frequency can be written as a complex quantity $\sigma_{0}=\sigma_{0}+\mathrm{j} \alpha$ where $\alpha^{-1}$ is the damping time constant, related to the wobble $\mathrm{Q}_{\mathrm{w}}$ (see below) as $\alpha=\sigma_{0} / 2 \mathrm{Q}_{\mathrm{w}}$. For $\mathrm{Q}=50-100$, as appears to be a likely result (Okubo, 1982b), the relaxation time constant is of the order 15-30 years: in the absence of excitation the Chandler wobble is reduced to $\mathrm{e}^{-1}$ of its value within this interval. This is not observed and estimates of $Q$ from the available record of about 80 years duration are therefore not only dependent on knowing the excitation mechanism but they are also based on an observational record whose duration is 
equal to a few relaxation time constants.

Very large earthquakes appear to be able to shift the inertia axis by amounts of $0 . " 01-0 . " 02$ (Mansinha et al. 1979) but there just do not appear to be enough of these large events to maintain the wobble against dissipation, even if the wobble $Q$ is 100 or 200 . The earthquakes with the largest seismic moments are the 1960 Chili and 1964 Alaska events and no comparable earthquakes have occurred since. Yet the wobble does not show evidence of major attenuation. Several authors have compared the polar motion records with the earthquake excitation series. Wilson and Gabay (1981), for example, do not find strong evidence for coherence between these two time series. More recently, Souriau and Cazenave (1985) concluded that the recent seismic excitation is wholly inadequate to generate the changes in Chandler wobble amplitude observed in recent years. The same conclusion is reached by Gross (1986) from an examination of a short (7 year) record for which precise pole positions were available. What is curious is that just as the accuracy of the polar motion data is increasing so are the estimates of seismic moments decreasing - at a time when global seismic instrumentation is improving. Estimates of seismic magnitudes do not, however, appear to show a particularly different distribution when viewed on time scales of a few decades. Are the older estimates of seismic moments too great? This subject has not progressed greatly since 1980. The question is still the same; if seismic excitation is important, can the missing power in the excitation function be generated by aseismic deformation, either as pre-or post-seismic changes in the inertia tensor. Observational evidence for this remains inadequate. Yet it is also unsatisfactory to use such an ill-constrained hypothesis to explain the excitation.

Atmospheric forcing of the Chandler wobble is somewhat more effective. Recent studies include those of Wahr (1983), Barnes et al. (1983) and Hide (1984). Wahr re-examined the combined atmospheric-oceanographic excitation function and concluded that the non-seasonal part is inadequate, by a factor of $2-3$, to excite the wobble, a conclusion not very different from that reached earlier by Wilson and Haubrich, (1976). Hide, however, argues that the atmospheric excitation may be adequate, although the computed excitation function is of short duration (about 4 years) and it is difficult to see how their excitation function can be tested to determine whether it can overcome the damping. In fact, I find it curious that damping does not enter into their discussion at all. What is obvious from these latter studies is that the atmospheric excitation function is sufficiently noisy to mask any turning points in the pole path that may be generated by earthquakes.

A third possible excitation mechanism is the action of electromagnetic torques arising from the penetration of the lower mantle by the time-dependent magnetic field generated within the liquid core (Runcorn, 1982). For such a mechanism to be effective, the changes in the magnetic field at the base of the mantle must be of short duration, a small fraction of the Chandler wobble period, but these would not be observed at the Earth's surface because of the screening effect of the electrical conductivity of the lower mantle. A simple observational test is therefore not possible. Runcorn suggests that the impulsive torque required to produce a change in the radius of the pole path of $0 . " 01$ is about equal to that required to produce the observed decade fluctuations recorded in the length-of-day (equal to about $5 \times 10^{-8}$ in $\mathrm{m}_{3}$ ). There is no apparent reason why these torques exhibit a preference to excite $m_{1}$ and $m_{2}$ rather than $m_{3}$ and the problem with this proposal is one of time-scale. First, the length-of-day spectrum has considerably less power at high frequencies than at low (Figure 3); second, the excitation of the high frequency changes in length-of-day is almost wholly due to 
changes in atmospheric angular momentum (see below). The mechanism proposed by Runcorn has to selectively produce impulsive torques about the $\mathrm{x}_{1}, \mathrm{x}_{2}$ axes only.

Dissipation. The third aspect of the Chandler wobble is the dissipation process: where is the wobble energy dissipated? Is it in the oceans or mantle, or is it at the core-mantle boundary? The last possibility is usually discarded because viscous and electromagnetic damping appear to be wholly inadequate and topographic coupling remains a poorly constrained mechanism. The dissipation is measured by the wobble $Q, Q_{w}$, defined as

$$
\mathrm{Q}_{\mathrm{w}}^{-1}=\frac{1}{2 \pi \mathrm{E}_{\mathrm{w}}} \int \frac{\mathrm{dE}}{\mathrm{dt}} \mathrm{dt}=\frac{1}{2 \pi} \frac{\Delta \mathrm{E}}{\mathrm{E}_{\mathrm{w}}}
$$

where $E_{w}$ is the total wobble energy stored in the Earth; the total energy that would, in the absence of any excitation, be dissipated in the time interval $0 \leqslant t \leqslant$ $\infty$. It includes the kinetic strain, gravitational and pole tide energies. $\Delta \mathrm{E}$ is the amount of energy dissipated in one cycle of the motion. It should be noted that $\mathrm{Q}_{\mathrm{w}}$ is not directly comparable with the shear mantle $Q, Q_{\mu}$, estimated from seismology and defined as

$$
\mathrm{Q}_{\mu}=\frac{1}{2 \pi} \frac{\Delta \mathrm{E}}{\mathrm{E}_{\mathrm{S}}}
$$

where $E_{S}$ is the strain energy. Smith and Dahlen (1981), Ben Menaham (1982) and Okubo (1982b) obtain $\mathrm{Q}_{\mathrm{w}} / \mathrm{Q}_{\mu} \simeq 1.5-2.1$ in contrast to earlier and apparently erroneous estimates of about 8-10 (Merriam and Lambeck, 1979; Stacey, 1969).

Estimates of $\mathrm{Q}_{\mathrm{w}}$ are uncertain for the reasons mentioned above but the consensus is still that $Q_{W}$ lies in the range of 50-100 despite occasional claims that $\mathrm{Q}_{\mathrm{w}}$ is very much higher. If dissipation occurs in the mantle, then $25 \leqslant \mathrm{Q}_{\mu} \leqslant 70$ compared with values of a few hundred obtained from low order seismic modes, consistent with models in which $\mathrm{Q}$ varies with frequency $\sigma$ according to $\sigma^{\alpha}$ with $0.05 \leqslant \alpha \leqslant 0.2$ (Smith and Dahlen, 1981; Molodenskii and Zharkov, 1982; Okubo, 1982b). This is consistent with the evidence based on the lengthening of the wobble period. This is perhaps the single most important result to have emerged in the last few years from the study of the Chandler wobble but it rests on the vexing assumption that the pole tide follows an equilibrium theory.

Most theoretical arguments favour an equilibrium theory for the pole tide whereas the observational evidence does not, there being strong evidence for enhanced amplification of this tide in some oceans. The theories remain, however, approximate, and the observational evidence rests on the assumption that a signal of a few mm amplitude at 1.2 years can be extracted globally from the sealevel records. Dickman (1985) has examined some of the observational evidence (see also Naito, 1979) and concludes that there is evidence for a world-wide enhancement of the pole tide. The departure from equilibrium need not be large to explain the damping of the wobble. If the analogy with the lunar tides is valid, the pole tide dissipation can be evaluated from the degree 2 , order 1 harmonic in the tide expansion. The observational data is entirely inadequate for estimating this component since a lag of this harmonic by only a few degrees is required for $\mathrm{Q}_{\mathrm{W}}=100$ (Lambeck, 1980, p.218).

If the above result for the frequency dependence of the mantle $Q$ is one of the important results of the past few years, the major open question remains the 
matter of dissipation, for if this occurs in the oceans, even if only in part, the result is no longer valid.

\section{Meteorological excitation}

It has been recognized for a number of years that the atmosphere forces the Earth's rotation, particularly the speed of rotation, over a broad range of periods, from about four years to as short as a few days and possibly less. In the case of changes in $\mathrm{m}_{3}$ or in length-of-day, the excitation is caused primarily by the zonal angular momentum exchange between the atmosphere and the solid Earth; $\psi$ (matter) $\approx 0.1 \psi($ motion). In the case of the polar motion it appears that the major excitation is caused by the redistribution of mass within the atmosphere and it is the $\Delta \mathbf{I}$ that have to be evaluated. Here the angular momentum contribution may, however, not be negligible if the winds depart from the geostrophic condition. The effect of winds on the Earth's rotation can be evaluated either through the functions $\psi$ (motion) or $\psi$ (torques). In the first case the requisite information is the wind velocity throughout the atmosphere while in the latter case the requisite information is the surface wind, the surface friction coefficient(s) and the detailed surface topography. The angular momentum approach is the simpler of the two, requiring fewer assumptions than the torque approach but the choice between the two usually reduces to one of availability of data: is it easier to evaluate wind velocity or to measure surface stress and mountain torques?

The atmospheric excitation functions could be viewed largely as nuisances that prevent the elucidation of otherwise more interesting geophysical phenomena. For example, as discussed above, the nature of the excitation of the Chandler wobble remains obscure. If atmospheric excitation is inadequate to maintain this oscillation, it does appear to make an overall contribution and other excitation mechanisms, such as earthquakes, need to be supplemented with the atmospheric part. The tidal terms in length-of-day are of geophysical interest in that they permit an evaluation of the combined solid Earth, core and ocean response to the tidal force at intermediate periods of about 13 and 27 days and 6 months. Atmospheric contributions to the excitations at these frequencies will degrade the result unless this can first be eliminated. Only if these corrections can be reliably made will it become possible to extend the data set for mantle attenuation over a broader frequency range. Another outstanding question is the excitation of the decade changes in length-of-day. It is not suggested here that the atmosphere plays a significant role but an important question is the nature of the mechanism responsible for these changes. For example, can the length-of-day time series or power spectrum be characterized by a series of impulse excitations or by a series of step or ramp functions? Such a distinction can be helpful in understanding the forcing functions (Lambeck, 1980, Chapter 4) but attempts to distinguish between such models have not been successful, in part because meteorological noise masks the details of the turning points or discontinuities in the length-of-day time series.

Length-of-day. Several analyses of the atmospheric excitation have been carried out since 1980. Lambeck and Hopgood (1982) examined the excitation of the length-of-day by zonal winds for 22 years from 1958 to 1980 . The data set comprised estimates of the zonal winds averaged over longitude and over monthly intervals and it was only possible to evaluate the excitation at frequencies up to about 6 cycles/year. The principal result was that the zonal winds dominate the excitation spectrum at periods up to about 50 months. Figure 4 illustrates the 

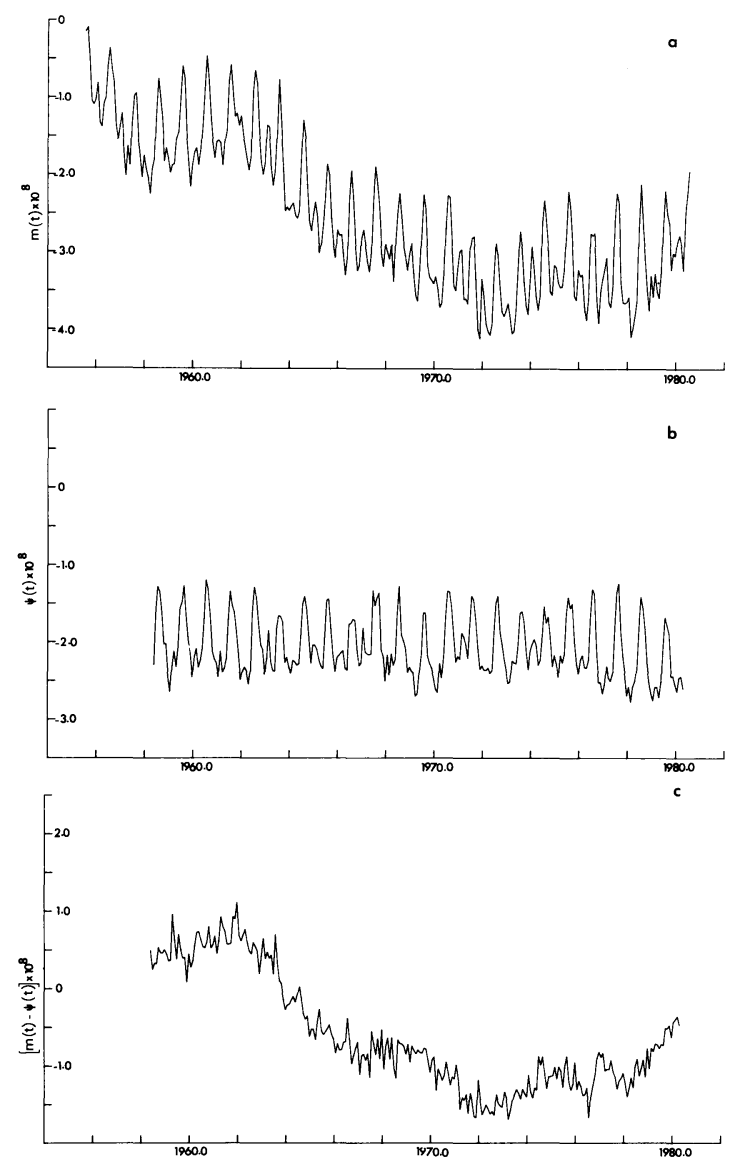

Figure 4. (a) Astronomical observations of the variations in the proportional change in the length-of-day $m_{3}(t)$ from 1955-1980. (b) Zonal wind excitation function $\psi_{3}(t)$ for a 22 year period. (c) $m_{3}(t)$ corrected for zonal winds and tides.

time series of $m_{3}$ and $\psi_{3}$ as well as the difference $m_{3}-\psi_{3}$. The last series contains residual high-frequency noise, attributable largely to inadequacies of the meteorological data but it does not exceed about $10^{-8}$, an order of magnitude less than the decade changes in $\mathrm{m}_{3}$ and inconsistent with the hypothesis that high frequency electromagnetic impulse torques have excited the Chandler motion during 
this 22 year period.

Rosen and Salstein (1983) evaluated the zonal wind excitation for a six year period with a three day resolution. Now the high frequency part of the spectrum can be examined and their excitation spectrum reveals several peaks in the 6-96 day range that rise above a continuum meteorological noise level. These include peaks near 17, 30 and 48 days (see also Langley et al., 1981; Morgan et al., 1985). This last term had already been noted in the length-of-day by Lambeck and Cazenave (1977) and attributed to atmospheric excitation. Enhanced energy in the spectrum also occurs about the $\mathbf{M}_{\mathrm{m}}$ and $\mathbf{M}_{\mathbf{f}}$ tidal frequencies. The important part of the study by Rosen and Salstein is that it demonstrates that the zonal wind excitation can be evaluated with considerable accuracy. They consider that $\psi_{3}$ may be systematically under-estimated by about $10 \%$ but that random errors, most important for evaluating the short-term changes, are believed to be only about $5 \%$. Limitations of their analysis includes the poor data distribution in southern latitudes (common to all analysis) and the restriction of the integration of zonal winds to the 100 mbar height. Winds above $100 \mathrm{mbars}$, including stratospheric winds, can make significant contributions to $\psi_{3}$ (Lambeck and Cazenave, 1973; Lambeck and Hopgood, 1981).

Polar motion. New analyses of the annual excitation of the polar motion have been made by Merriam (1982) and Wahr (1983). Both studies confirm the major role played by the redistribution of atmospheric mass in exciting the seasonal changes in the Earth's wobble. The two studies also emphasize the importance of adequately treating the ocean response to changes in surface pressure. This is the inverted barometer problem raised by Munk and MacDonald (1960) and the atmospheric and oceanic loading problems are closely coupled. A major uncertainty in estimating the total seasonal excitation is the contribution arising from the seasonal changes in ground and surface water. All analyses use a result of Van Hylckama (1970) but a re-evaluation of this is urgently needed. A preliminary step in this direction has been taken by Wilson and Hinnov (1985) who have evaluated the northern hemisphere water storage excitation for the years 1900-1979 using a simple hydrological model and only observations of surface temperature and pressure. The absence of the southern hemisphere makes it impossible to comment on the magnitude of this excitation, but what is of interest is that Wilson and Hinnov find that the spectrum of partial excitation is fairly broad about the annual frequency and that it may contribute to the excitation of the Chandler wobble.

Another source of uncertainty has been the evaluation of $\psi_{i}$ (motion) for $i=1,2$. If the atmospheric circulation follows the geostrophic condition $\psi_{i}$ vanishes (Munk and MacDonald, 1960), and the meridional winds do not contribute. It has generally been impossible to test this assumption because observational evidence on the meridional winds has been inadequate to evaluate $h_{1}$ and $h_{2}$. Wilson and Haubrich (1976) evaluated the contribution to $\psi$ (torque) arising from mountain torques only, argueing that surface friction is negligible. But it does appear that surface torques are likely to be at least as important, if not more so, in establishing the overall angular momentum transfer between the atmosphere and the solid earth (Newton, 1971; Wahr and Oort, 1984). Wahr (1983) concludes, not unexpectedly, that the mountain and surface friction torque excitations are not well determined but that they each represent less than about $10 \%$ of the pressure contribution $\psi$ (matter). 
High frequency atmospheric excitation functions. Major efforts are underway at several centres to systematically compute the atmospheric excitation functions, both $\psi_{\mathrm{i}}$ (matter) and $\psi_{\mathrm{i}}$ (motion), on a daily basis (see the papers by Whysall et al., 1985; Naito and Yokoyama, 1985; Salstein and Rosen, 1985). A comparison of some of these data sets has been made by Eubanks et al. (1985) and this shows that there is considerable consistency between the various solutions for $\psi_{i}$ such that the atmospheric contributions can be removed with some confidence that the residual excitation or rotation change has geophysical meaning: a state that had not been reached by 1980 . Particularly important is the high frequency noise-like component in the polar motion excitation spectrum (Barnes et al. 1983). Without corrections for this meteorological noise, high precision and high resolution observations of the Earth's rotation loose much of their interest. It is regretable, to Earth rotationists at least, that there has been a dirth of large earthquakes in the few years for which this meteorological excitation has been available.

\section{Tidal changes in length-of-day}

The dominant tidal effect in the Earth's rotation is the secular change in the length of day produced by the dissipation of the tides raised in the oceans and, to a much lesser degree, in the solid Earth. The problem of this tidal dissipation and the associated dynamical evolution of the Earth-Moon system is not one upon which I wish to dwell here (see, instead, Lambeck, 1980; and papers in Brosche and Sundermann, 1982). Other tidal effects are the periodic changes in the length-of-day, of six month (SSa), and $27(\mathrm{Mm})$ and 13 (Mf) day periods. The first of these is coincident with the somewhat larger amplitude semi-annual atmospheric excitation and this latter will need to be known with very high precision for the solid tide result to provide useful geophysical information. The $\mathrm{Mm}$ and $\mathrm{Mf}$ tides are of greater interest, in particularly for estimating the frequency dependence of $Q$.

Numerous analyses of the tidal Love number $k$ have been made but no consistent result appears. For example, Guinot (1974) found that $k_{M f}>k_{M m}$. Others, for example Yoder et al. (1981), found that $\mathrm{k}_{\mathrm{Mf}} \simeq \mathrm{k}_{\mathrm{Mm}}$. Capitaine and Guinot (1985) noted that $\mathrm{k}_{\mathrm{Mf}}$ exhibits considerable variability with time whereas $\mathrm{k}_{\mathrm{Mm}}$ appeared to be relatively stable. This behaviour led to the suggestion by Lambeck and Cazenave (1974) that, while there is no evidence for a significant signal of exactly tidal frequency in the atmospheric excitation, there is an increase in the meteorological noise near these tidal frequencies. The results of Rosen and Salstein (1983) confirm this. These results, and subsequent ones by Salstein and Rosen (1985), indicate that the length-of-day observations can, and should, be first corrected for this atmospheric excitation before tidal Love numbers are estimated. Merriam (1984) notes, however, that some of these different results are also a consequence of the use of Woolard's nutation series instead of the improved Wahr (1981) theory although the Capitaine and Guinot results are referenced to the latter theory and the problem remains.

Any complete theory of the earth's tidal deformations has to take into account the ocean tide's response and its loading of the solid Earth and the degree of coupling, or lack of it, between the mantle and core. Also required is a precise description of the motion of the tide-raising body about the Earth. Recent discussions of these theories are by Merriam (1980), Wahr et al. (1981) and Yoder et al. (1981) and the agreement between them appears to be satisfactory at the 
present levels of precision. An assumption in these theories does have to be made about whether or not these tides follow an equilibrium theory but while this point has not yet been resolved it does not appear to be of a major consequence (Merriam, 1984).

Merriam (1984) appears to be the only one who has corrected the length-of-day data for the atmospheric excitation and finds that $\mathrm{k}_{\mathrm{Mf}}$ equals $\mathrm{k}_{\mathrm{Mm}}$ within the observational errors. Equally important is that the statistics of the atmospherically corrected results are better than those of the analyses of the uncorrected length-of-day data set. The average value for $k$ found is, however, marginally greater than that predicted at higher frequencies from seismological observation, by an amount that is consistent with the frequency dependent $Q$ law with $\alpha$ equal to about 0.2-0.3. (A similar value was found by Lambeck and Nakiboglu (1983) from an examination of the tidal response at 18.6 years albethis an equally provisional result). The error bars are still sufficiently large to permit a wide range of $\alpha$ value from 0 to more than 0.3 , for the result is based on only a 5 year interval for which both the atmospheric excitation and the corrected astronomical estimates of length-of-day were available. Both data sets have subsequently been extended and a new analysis is worthwhile.

\section{The nearly diurnal free core motion}

The solid mantle-fluid core system possesses two free modes; the Chandler wobble and a free core mode. The latter has a frequency of approximately $\sigma \simeq-\omega_{0}\left(1+f_{c} A / A_{m}\right)$ where $A$ and $A_{m}$ are the moments of inertia of the whole Earth and mantle respectively and $f_{c}$ is the flattening of the core. That is, referenced to the earth-fixed axes, the oscillation is nearly diurnal, but retrograde, differing by a few minutes from a sidereal day. Expressed relative to inertial, space fixed axes this retrograde motion is of long period (eqn 7), its frequency $\sigma^{-}$ being

$$
\sigma^{\prime}=\sigma+\omega_{0} \simeq-\mathrm{f}_{\mathrm{c}} \omega_{0} \mathrm{~A} / \mathrm{A}_{\mathrm{m}} .
$$

These estimates are for a rigid mantle and a perfectly fluid core of ellipsoidal shape. More rigorous theories yield a retrograde motion in space with a period of about 460 days (Toomre, 1974).

Being a free mode, the amplitude of this oscillation is arbitrary, a function of the competing processes of excitation and dissipation. Numerous attempts have been made to search for this term in observations of polar motion or variations in astronomical latitude but, as emphasized by Toomre (1974), if such a term exists, its amplitude in the inertial reference frame will be some 460 times as great and the place to search for it is in the nutation data. But here, also, it was not found (e.g. Rochester et al. 1974), which suggests that the amplitude is very small indeed. The only hope of detecting this free core mode, if it exists at all, lies in the examination of some of the recently revised high precision and consistent optical astronomy data sets or in the high precision long baseline radio interferometry observations of the Earth's nutation. Capitaine and Xiao (1982) examined 14 years of Bureau International de l'Heure observations of pole positions and universal time and noted a retrograde oscillation, referenced to inertial axes, of period of 434-444 days that could be attributed to the free core mode. Robertson et al. (1986), using the observations of the International Radio Interferometric Surveying network for a very short time interval from January 
1984 to April 1985, detected a small amplitude oscillation in the frequency range of 0.73-0.91 cycles per year (400-500 day period) but this result was subsequently withdrawn. No doubt we will hear more about this subject in the future.

\section{Conclusion}

I have been able to discuss here only a few aspects of the Earth's rotation. I have not mentioned the forced nutations and the important new observational data that is becoming available for these motions. I have not mentioned, other than in passing, the decade-scale changes in length-of-day and their relation with changes in the Earth's magnetic field. Rochester (1984) touched upon this in his review and I cannot add to the subject.

There has been much progress in understanding the Earth's rotation since about 1980. In particular, there is a better understanding of the theory of the rotation of an anelastic body with a fluid core, only partly covered by oceans and with an atmosphere. Some of the outstanding problems of 1980 have not, however, made much progress. The excitation and dissipation of the Chandler wobble remain unsolved problems. Here the answers will probably have to wait for lengthy new data sets of the Earth's rotation, as well as for improved geophysical (including oceanographic and meteorological) data and theory. We can expect many more papers on this subject.

Probably the single most important change of the past five or so years has been the delivery of a long awaited promise, of new high precision, high resolution results for the Earth's rotation, made nearly 20 years ago (MacDonald, 1967; Gold, 1967). Such is the progress of science and technology. But these intervening years have been well spent in producing theories to match these new observations. Undoubtedly there will be many surprises as the new data sets are extended.

\section{Refrences}

Barnes, R.T.H., R. Hide, A.A. White, and C.A. Wilson, 1983. Atmospheric angular momentum fluctuations, length-of-day changes and polar motion. Proc. Roy. Soc. Lond., A 387, 31-73.

Ben-Menahem, A., 1982. A new derivation of the ratio Q(wobble)/Q(mantle). Geophys. J., 70, 535-537.

Brosche, P. and J., Sundermann, 1982. Tidal Friction and the Earth's Rotation II, Springer-Verlag, Berlin, $345 \mathrm{pp}$.

Capitaine N., and N. Xiao, 1982. Some terms of nutation derived from the BIH data. Geophys. J., 68, 805-814.

Capitaine, N., and B. Guinot, 1985. Anomalies of some tidal wave, of UT1. Geophys. J., 81, 563-568.

Carter, W.E., 1982. Refinements of the polar motion frequency modulation hypothesis. J. Geophys. Res., 87, 7025-7028.

Carton, J.A., and J.M. Wahr, 1986. Modelling the pole tide and its effect on the Earth's rotation. Geophys. J., 84, 121-137.

Chao, B.F. 1983. Autoregressive harmonic analysis of the Earth's polar motion using homogeneous international latitude service data. J. Geophys. Res., 88, 10299-10307 
Colombo, G., and I. Shapiro, (1968). Theoretical model for the Chandler wobble. Nature, 217, 156-157.

Dickman, S.R., 1985. The self-consistent dynamic pole tide in the global oceans. Geophys. J., 81, 157-174.

Dickman, S.R., and D.J. Steinberg, 1986. New aspects of the equilibrium pole tide. Geophys. J., 86, 515-529.

Eubanks, T.M., J.A. Steppe and J.O. Dickey, 1985. The atmospheric excitation of Earth orientation changes during Merit. Proc. Int. Conf. Earth rotation and their terrestrial frame. Dept. Geod. Science, Ohio State University, pp 469-483.

Gold, T., 1967. Radio method for the precise measurement of the rotation period of the Earth. Science, 157, 302-304.

Gross, R.S., 1986. The influence of earthquakes on the Chandler wobble during 1977-1983. Geophys. J., 85, 161-177.

Guinot, B., 1974. A determination of the Love number $k$ from the periodic waves of UT1. Astron. Astrophys., 36, 1-4.

Hide, R., 1984. Rotation of the atmospheres of the Earth and planets. Phil. Trans. R. Soc Lond., A 313, 107-121.

Jeffreys, H., 1940. The variation of latitude. Mon. Not. R. Astron. Soc., 100, 139-155

Jeffreys, H., 1968. The variation of latitude. Mon. Not. R. Astr. Soc., 141, 255-268.

Jeffreys, H. 1970. The Earth (fifth edition). Cambridge University Press, 525pp.

Lambeck, K., 1980. The Earth's Variable Rotation. Cambridge University Press, $449 p$.

Lambeck, K., 1984. Hula dancers, Walter Munk and the rotation of the Earth. In A Celebration in Geophysics and Oceanography - 1982 : In Honor of Walter Munk. Scripps Institution of Oceanography Reference Series 84-5.pp 87-95.

Lambeck, K., 1986. Banded iron formations. Nature, 320, 547.

Lambeck, K., and A. Cazenave, 1974. The Earth's rotation and atmospheric circulation-II. The continuum. Geophys. J., 38, 49-61.

Lambeck, K., and A. Cazenave, 1977. The Earth's variable rotation: a discussion of some meteorological and oceanic causes and consequences. Phil. Trans. R. Soc. Lond., A 284, 495-506.

Lambeck, K., and P. Hopgood, 1981. The Earth's rotation and atmospheric circulation, from 1963 to 1973. Geophys. J., 64, 67-89.

Lambeck, K., and P. Hopgood, 1982. The Earth's rotation and atmospheric circulation: 1958-1980. Geophys. J., 71, 581-587.

Lambeck, K., and S.M. Nakiboglu, 1983. Long-period Love numbers and their frequency dependence due to dispersion effects. Geophys. Res. Lett., $10,857-860$.

MacDonald, G.J.F., 1967. Implications for geophysics of the precise measurement of the Earth's rotation. Science, 157, 304-307.

Mansinha, L., D.E. Smylie, and C.H. Chapman, (1979). Seismic excitations of the Chandler wobble revisited. Geophys. J., 59, 1-17.

Masters, G., 1979. Observational constraints on the chemical and thermal structure of the Earth's deep interior. Geophys. J., 57, 507-534.

Merriam, J.B., 1982. Meteorological excitation of the annual polar motion. Geophys. J., 70, 41-56.

Merriam, J.B., 1983. Variations in the Earth's rotation. Sci. Prog., 68, 387-401. 
Merriam, J.B., 1984. Tidal terms in universal time: effects of zonal winds and mantle Q. J. Geophys. Res., 89, 10109-10114.

Merriam, J.B., and K. Lambeck, 1979. Comments on the Chandler wobble Q. Geophys. J., 59, 281-286.

Molodenskii, S.M. and V.N. Zharkov, 1982. Chandler Wobble and frequency dependence of $\mathrm{Q}$ of the Earth's mantle. Phys. Solid Earth, 18, 245-254.

Munk, W.H. and G.J.F. MacDonald, 1960. The Rotation of the Earth. Cambridge University Press. 323pp.

Naito, I., 1979. Effects of the pole tide on the Chandler wobble. J. Phys. Earth, 27, 7-20.

Naito, I., and K. Yokoyama 1985. A computation of atmospheric excitations functions for the Earth's rotation based on J.M.A. global analysis data. Proc. Int. Conf. Earth Rotation and their Terrestrial Frame. Dept. Geod. Science, Ohio State University, pp 434-439.

Nakiboglu, S.M. and K. Lambeck, 1980. Deglaciation effects on the rotation of the Earth. Geophys. J., 62, 49-58.

Newton, C.W., 1971. Mountain torques in the global angular momentum balance. $J$. Atmos. Sci., 28, 623-628.

O'Connor, W.P., 1986. On the applicaiton of asymptotic analysis to the dynamical theory of the pole tide. Geophys. J., 85, 1-11.

Okubo, S., 1982a. Is the Chandler period variable? Geophys. J., 71, 629-649.

Okubo, S., 1982b. Theoretical and observed Q of the Chandler wobble Love number approach. Geophys. J., 71, 647-657.

Pederson, G.P.H. and M.G. Rochester 1972. Spectral analysis of the chandler wobble. In Rotation of the Earth (P. Melchior and S.Yumi, eds.) D. Reidel, Dordrecht, pp 33-38.

Proverbio, E., F. Carta and F. Mazzoleni, 1972. In Rotation of the Earth (P. Melchior and S. Yumi, eds.) D Reidel, Dordrecht 43-45.

Robertson, D.S., W.E. Carter and J.M. Wahr, 1986. Possible detection of the Earth's free-core nutation. Geophys. Res. Lett., 13, 949-952.

Rochester, M.G., 1984. Causes of fluctuations in the rotation of the Earth. Phil. Trans. R. Soc. Lond. A 313, 95-105

Rochester, M.G., O.G. Jensen and D.E. Smylie, 1974. A search for the Earth's nearly diurnal free wobble. Geophys. J., 38, 349-363.

Rosen, R.D., and D.A. Salstein, 1983. Variations in atmospheric angular momentum on global and regional scales and the length of day. $J$. Geophys. Res., 88, 5451-5470.

Salstein, D.A., and R.D. Rosen, 1985. Computations of atmospheric angular momentum, with emphasis on the MERIT period. Proc. Int. Conf. Earth Rotation and their Terrestrial Frame. Dept. Geod. Science, Ohio State University, pp 440-449.

Smith, M.L., 1977. Wobble and nutation of the Earth. Geophys. J., 50, 103-140.

Smith, M.L. and F.A. Dahlen, 1981. The period and Q of the Chandler wobble. Geophys. J., 64, 223-281.

Stacey, F.D., 1969. Physics of the Earth. John Wiley, New York, 324pp.

Souriau, A., and A. Cazenave, 1985. Reevaluation of the Chandler wobble seismic excitation from recent data. Earth Planet. Sci. Lett., 75, 410-416.

Toomre, A., 1974. On the "nearly diurnal wobble" of the Earth. Geophys. J., 38, 335-348. 
Van Hykkama, T.E.A., 1970. Water balance and Earth unbalance. Publ. 92. Int. Ass. Sci. Hydr., 434-553.

Wahr, J.M., 1981. Forced nutations of an elliptical rotating, elastic and oceanless Earth. Geophys. J., 64, 705-727.

Wahr, J.M., 1982. The effects of the atmosphere and oceans on the Earth's wobble - I. Theory. Geophys. J., 70, 349-372.

Wahr, J.M., 1983. The effects of the atmosphere and oceans on the Earth's wobble and on the seasonal variations in the length of day - II. Results. Geophys. J., 74, 451-487.

Wahr, J.M., 1985. The Earth's rotation rate. American Scientist, 73, 41-46.

Wahr, J.M., and A.H. Oort. Friction and mountain torque estimates from global atmospheric data. J. Atmos. Sci, 41, 190-204.

Wahr, J.M., T. Sasao, and M.L, Smith, 1981. Effect of the fluid core on changes on the length of day due to long period tides, 1 Geophys. J., 64, 635-650.

Walker, J.C.G., and K.J. Zahnle, 1986. Lunar nodal tide and distance to the Moon during the Precambrian. Nature, 320, 600-602.

Wilson, C.R., and R.A. Haubrich, 1976. Meteorological excitation of the Earth's wobble. Geophys. J., 46, 707-743.

Wilson, C.R., and S. Gabay, 1981. Excitation of the Earth's polar motion: a reassessment with new data. Geophys. Res. Lett., 8, 745-748.

Wilson, C.R. and L. Hinnov. 1985. Water storage effects on the Earth's rotation. Proc. Int. Conf. Earth Rotation and their Terrestrial Reference Frame. Dept. Geod. Science, Ohio State University, pp 417-433.

Wilson, C.R., and R.O. Vicente, 1980. An analysis of the homogeneous ILS polar motion series. Geophys. J., 62, 605-616.

Whysall, K.D.B., R. Hide and M.S. Bell current work on the Earth's rotation at the United Kingdom Meteorological Office. Proc. Int. Conf. Earth Rotation and their Terrestrial Reference Frame. Dept. Geod. Science, Ohio State University, pp 417-433.

Wu, P., and W.R. Peltier, 1984. Pleistocene deglaciation and the Earth's rotation: a new analysis. Geophys. J., 76, 753-791.

Yoder, C.F., J.G. Williams and M.E. Parke, 1981. Tidal variations of earth rotation. J. Geophys. Res., 86, 881-889.

Yuen, D.A., R. Sabadini, and E. Boschi, 1982. The viscosity of the lower mantle as inferred from rotational data. J. Geophys. Res., 87, 10745-10762. 